\title{
Study on Triaxial Compression Creep Test and Numerical Simulation of Jurassic Sandstone in Northern Shaanxi
}

\author{
Zhipei Zhang, Qi Men and Yi Cheng \\ Xian University of Science and Technology College of Geology \& Environment, Xi'an, Shaanxi 710054, China
}

\begin{abstract}
Based on the creep characteristics of the overlying Jurassic sandstone in the goaf in Yulin area of Northern Shaanxiby the RLW-2000 microcomputer controlled rock triaxial compression creep testing machine, the parameter values of Burgers creep model of the Jurassic sandstone are determined, and the creep characteristics of Jurassic sandstone are described. The parameter values of Burgers creep model of the Jurassic sandstone about FLAC3D simulation are determined by using the method of FLAC3D simulation and laboratory test, the simulated results provide the basis for long term stability evaluation by using computer simulation.
\end{abstract}

Keywords-Yulin area of Northern Shaanxi; Jurassic sandstone; triaxial compression creep test; Burgers model

\section{INTRODUCTION}

Yulin of Shaanxi province exists a venue mined-out area, the area contains the Jurassic middle Zhiluo Fm green medium grained sandstone, the geological structure is relative simple. The rock dip Angle commonly $2 \sim 5^{\circ}$,its physical and mechanical properties of rocks are follows:the density of 2.35 $\mathrm{t} / \mathrm{m} 3$, dry uniaxial compressive strength of $56.3 \mathrm{MPa}$, Saturated uniaxial compressive strength $32.7 \mathrm{MPa}$, softening coefficient of 0.38 , dry modulus of $1.213 \times 104 \mathrm{MPa}$, poisson's ratio of 0.29 , cohesion is 3.82 MPa. In recent years, there are many researchs in the process of sandstone creep properties study. For the purpose of research the problem of the long-term stability of the mined-out area, in this paper, the sandstone triaxial compression creep property was tested under different confining pressure, and ensures the Burgers model parameters, and put forward the FLAC3D to simulate calculation parameters, the creep properties of sandstone of shaanxi research has certain theoretical and engineering significance ${ }^{[2-9]}$.

\section{TRIAXIAL COMPRESSION CREEP TEST}

Creep test use the RLW - 2000 microcomputer rock triaxial creep testing machine. the sample by drilled taken from a mined-out area which contains Jurassic medium coarse sandstone in Yulin city. The cylinder standard specimen, size of $\varphi 50 \times 110 \mathrm{~mm}$. The top and bottom surface is flat and level, parallel degree is less than $0.02 \mathrm{~mm}$, in order to prevent the condition of stress concentration have effect on the test results in the experimental process. Sandstone are mainly distributed $40 \mathrm{~m}-80 \mathrm{~m}$ under the ground which overlaid by the quaternary system, and the sample taken deep in the $50 \mathrm{~m}$, to simulate the axial pressure of deep in the $50 \mathrm{~m}$, the overlying rock and soil layer average density of $20 \mathrm{~N} / \mathrm{m}^{3}$, after calculation, the axial loading force test of $20 \mathrm{KN}$ (10.19 $\mathrm{MPa})$, confining pressure according to proportional relationship is $1 \mathrm{MPa}, 2 \mathrm{MPa}, 4 \mathrm{MPa}$, $5 \mathrm{MPa}$. To load the first $1 \mathrm{MPa}$ confining pressure every $45 \mathrm{~h}$ 50h confining pressure add $1 \mathrm{MPa}$, until the confining pressure is loaded into the $5 \mathrm{MPa}$, a total of $190 \mathrm{~h}$ test time. in the test process, the temperature constant at about $20{ }^{\circ} \mathrm{C}$ and computer automatically collecting data such as load, deformation, strain and time.

\section{TRIAXIAL COMPRESSION CREEP TEST RESULTS ANALYSIS}

By creep curve (FIG.1-FIG.4) can be obtained: a) The curve gradually changes from plain tilt to gentle as increase of load. The specimen is given priority to with instantaneous displacement When the stress is small, its creep deformation increase and then gradually achieve stability as time goes by; creep variable increased With the increase of stress; And continue to increase over time, the deformation at a certain creep rate increased steadily, the curve is smooth, the stage for the constant creep stage;

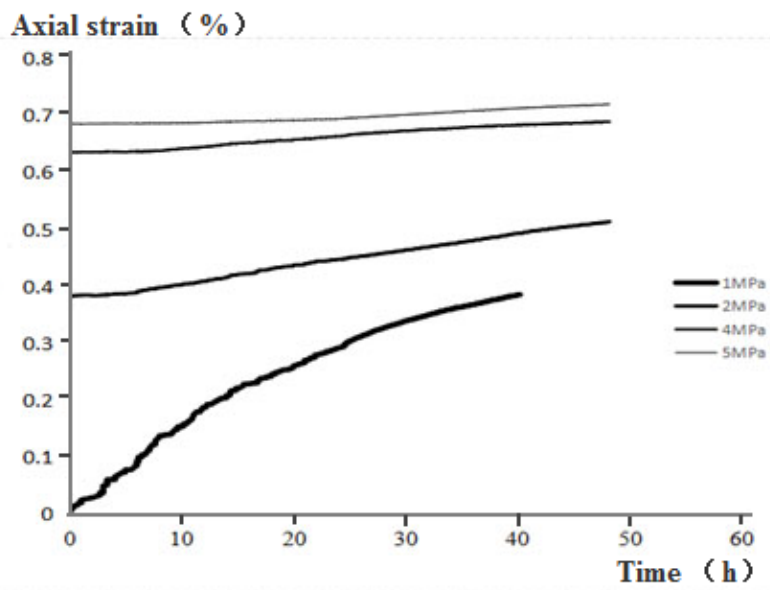

FIGURE I. AXIAL STRAIN- TIME CURVES OF 20KN UNDER DIFFERENT CONFINING PRESSURES 


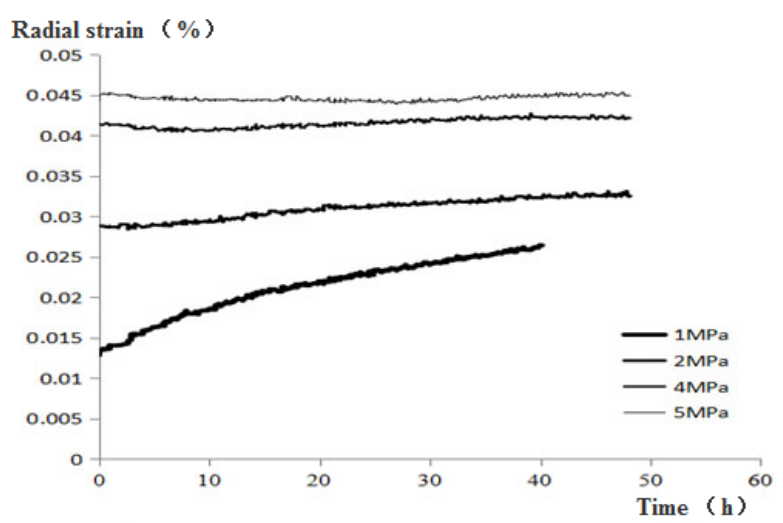

FIGURE II. RADIAL STRAIN- TIME CURVES OF 20KN UNDER DIFFERENT CONFINING PRESSURES

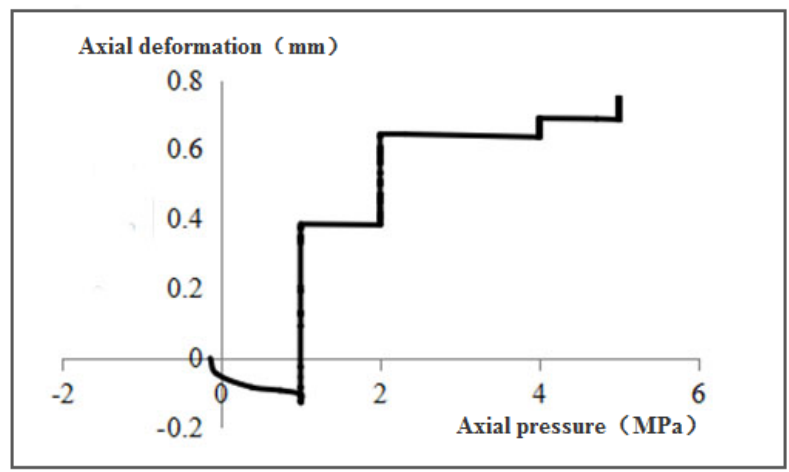

FIGURE III. AXIAL STRAIN TIME CURVES OF 20KN UNDER DIFFERENT CONFINING PRESSURES

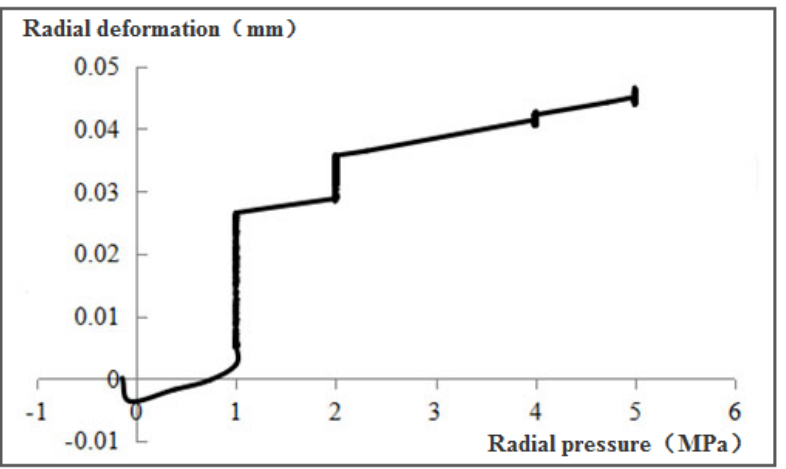

FIGURE IV. RADIAL STRAIN CURVES OF 20KN UNDER DIFFERENT CONFINING PRESSURES

b) after the instantaneous applied axial stress $\sigma$, rock immediately produce the instantaneous elastic deformation Moreover, the instantaneous deformation become smaller as increase the radial pressure, the curve is smooth. we can know that the deformation slowly increase with increase of time from curve.

c) when the confining pressure is equal to $1 \mathrm{MPa}$, axial creep value is greater than the confine creep value and curve is obvious diagonals. When the confining pressure is set to $2 \mathrm{MPa}$, curve for slightly sloping curve. When the confining pressure of $4 \mathrm{MPa}, 5 \mathrm{MPa}$, the curve for a little smooth curve. General characteristics as follows: with the increase of confining pressure, axial creep value increase, whereas relatively transient value is bigger, the lateral creep value and the ratio of lateral transient value, is relatively large.

\section{CONSTITUTIVE EQUATION AND THE DETERMINATION OF CREEP PARAMETERS}

\section{A. The Determination of Creep Constant}

Creep curve is obtained by triaxial creep test in instantaneous deformation (FIG. 5) first, and then after the initial stage of creep into the stable creep stage. Burgers model was used to describe it creep properties list the creep equation of Burgers model first [1] :

$$
\begin{aligned}
\varepsilon_{1}(t)=\frac{\sigma_{1}-\sigma_{2}}{3 G_{0}}+ & \frac{\sigma_{1}+2 \sigma_{2}}{9 K}+ \\
& \frac{\sigma_{1}-\sigma_{2}}{3}\left[\frac{\mathrm{t}}{\eta_{2}}+\frac{1}{G_{1}}\left(1-e^{-\frac{G_{1}}{\eta_{1}}}\right)\right]
\end{aligned}
$$

change to:

$$
\varepsilon(\mathrm{t})=A+B t+C\left(1-e^{-D t}\right)
$$

Noted:

$$
\begin{gathered}
A=\frac{\sigma_{1}+\sigma_{2}}{3 G_{0}}+\frac{\sigma_{1}+2 \sigma_{2}}{9 K} \quad B=\frac{\sigma_{1}-\sigma_{2}}{3 \eta_{2}} \\
C=\frac{\sigma_{1}-\sigma_{2}}{3 G_{1}}, D=\frac{G_{1}}{\eta_{1}}
\end{gathered}
$$

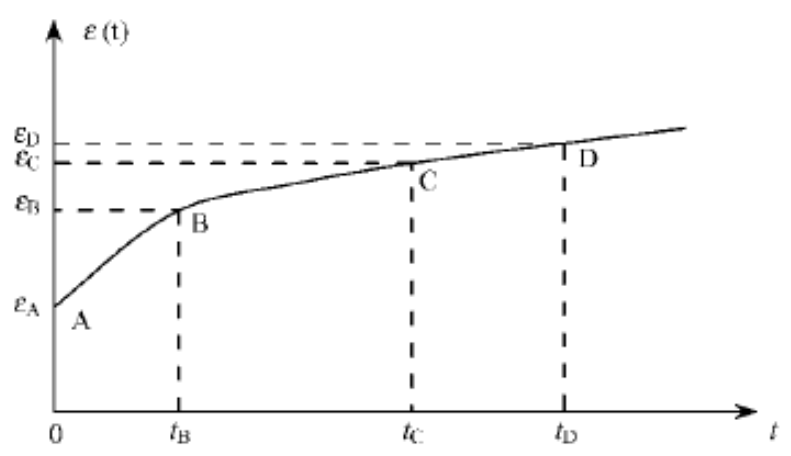

FIGURE V. CREEP CURVE

Instantaneous deformation on the creep curve, select the end, the initial creep stage in $\mathrm{B}$, stable creep stage in $\mathrm{C}$ and $\mathrm{D}$, available: $\varepsilon_{A}, 0 ; \varepsilon_{B}, \mathrm{t}_{B} ; \varepsilon_{C}, \mathrm{t}_{\mathrm{C}} ; \varepsilon_{\mathrm{D}}, \mathrm{t}_{\mathrm{D}}$ So the type (2) of $\mathrm{A}, \mathrm{B}, \mathrm{C}, \mathrm{D}$ can be determined by the type:

$$
\begin{aligned}
& \mathrm{A}=\varepsilon_{\mathrm{A}}, \mathrm{B}=\frac{\varepsilon_{\mathrm{D}}-\varepsilon_{C}}{t_{D}-t_{C}} \\
& \mathrm{C}=\varepsilon_{\mathrm{D}}-\mathrm{A}-\mathrm{Bt}_{0}
\end{aligned}
$$




$$
\mathrm{D}=-\frac{1}{t_{B}} \ln \left(1-\frac{\varepsilon_{B}-A-B t_{B}}{C}\right)
$$

Creep constants in the model, $G_{0}, G_{1}, \eta_{1}, \eta_{2}$ are given by type (3)

$$
\begin{aligned}
& G_{0}=\frac{3 K\left(\sigma_{1}-\sigma_{2}\right)}{9 A K-\left(\sigma_{1}+2 \sigma_{2}\right)}, G_{1}=\frac{\sigma_{1}-\sigma_{2}}{3 C}, \\
& \eta_{1}=\frac{G_{1}}{D}, \eta_{2}=\frac{\sigma_{1}-\sigma_{2}}{3 B}
\end{aligned}
$$

\section{B. Creep Mechanics Parameters of the Model}

According to the type (4) the rheological constitutive equation, the use of mathematical software Origin8.0 nonlinear fitting function of siltstone triaxial creep test to fitting the measured data, calculate A, B, C, D values, as shown in figure 6 , for in the axial pressure is equal to $20 \mathrm{KN}$, confining pressure is equal to $1 \mathrm{MPa}$ time and curve fitting of experimental data.The experimental creep equation parameters are shown in table 1.

TABLE I. CREEP PARAMETERS OF THE SPECIMENS IN THE THREE AXIAL LOADING TEST

\begin{tabular}{cccccc}
\hline $\begin{array}{c}\text { load } \\
\text { MPa }\end{array}$ & $\begin{array}{c}\mathrm{G}_{0} \\
\mathrm{MPa}\end{array}$ & $\begin{array}{c}\eta_{1} \\
\mathrm{MPa} \cdot \mathrm{h}\end{array}$ & $\begin{array}{c}\mathrm{G}_{1} \\
\mathrm{MPa}\end{array}$ & $\begin{array}{c}\eta_{2} \\
\mathrm{MPa} \cdot \mathrm{h}\end{array}$ & $\begin{array}{c}\mathrm{K} \\
\mathrm{MPa}\end{array}$ \\
\hline 1 & 84861 & 9767290 & 1019412 & 33322640 & 31818 \\
2 & 108964 & 5168960 & 3219290 & 66040540 & 31818 \\
4 & 141015 & 1352670 & 4676840 & 13351810 & 31818 \\
5 & 152842 & 13351810 & 904973 & 55430400 & 31818 \\
\hline
\end{tabular}

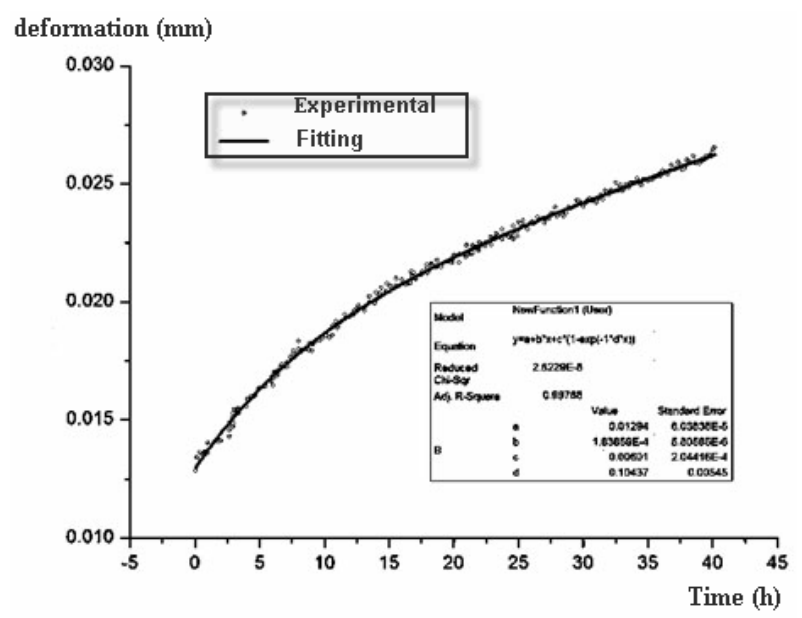

FIGURE VI. CREEP TEST AND REGRESSION CURVE

\section{TRIAXIAL COMPRESSION CREEP TEST OF SIMULATION RESEARCH}

Using FLAC3D to triaxial compression test of sandstone process computer simulation ${ }^{[1]}$, the model size of numerical simulation is same as cylinder which triaxial compression test used for. The Size diameter is $50 \mathrm{~mm}$ and high is $110 \mathrm{~mm}$, There are 2200 units and 2412 nodes in model. Select the Burgers model. Calculation scheme for the axial compression is equal to $20 \mathrm{KN}$, confining pressure is set to $1 \mathrm{MPa}$, pressing time of $50 \mathrm{~h}$; Confining pressure added to $2 \mathrm{MPa}$ is $100 \mathrm{~h}$; 4 $\mathrm{MPa}$ is $50 \mathrm{~h}, 5 \mathrm{MPa}$ is $70 \mathrm{~h}$. The total pressure time of $270 \mathrm{~h}$. As shown in figure 7, fitting curve for the numerical simulation and experiment, after $70 \mathrm{~h}$, the model of axial strain reached a balance, deformation maintained within $0.3 \mathrm{~mm}$, appeared a certain amount of rebound, it is mainly the effect of confining pressure. After that, add further confining pressure until it reached $4 \mathrm{MPa}$, deformation creates a greater change due to the confining pressure is directly added $2 \mathrm{MPa}$, curve obviously springback is more obvious than before, this is because the model after a certain time of the loading has been deformation tends to be stable, thus it can be seen that the increase of confining pressure increases the strength of the rock, so it lead to the decline of the deformation. then, the confining pressure loading by $5 \mathrm{MPa}$, only have a small rebound and deformation, it shows that in $20 \mathrm{KN}$ (10.19 MPa) under axial compression, the deformation of the rock get stability, continue to increase in confining pressure, the deformation will not have big change.

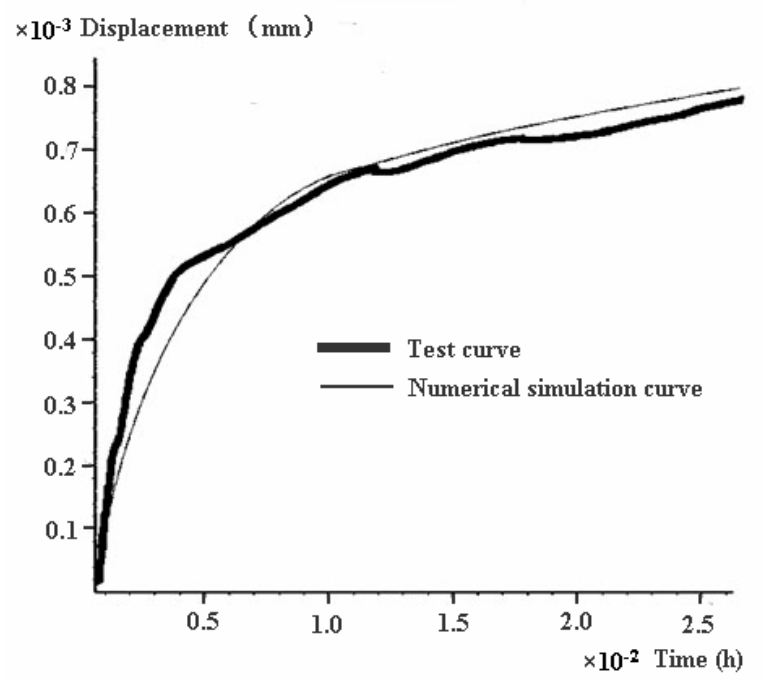

FIGURE VII. COMPARISON OF AXIAL DISPLACEMENT AND NUMERICAL SIMULATION OF AXIAL DISPLACEMENT

Burgers was used through simulation calculation, the model parameters are: $1.29 * 10^{9} \mathrm{~Pa}$ volume modulus, shear modulus of $8.3 * 108 \mathrm{~Pa}, 8 \times 106 \mathrm{~Pa}$ cohesion, internal friction Angle is $30.5^{\circ}$, the tensile strength of $2.57 * 10^{7} \mathrm{~Pa}$, density of 2600 $\mathrm{kg} / \mathrm{m}^{3}$, G1 is $8.4 * 10^{4} \mathrm{MPa}, \eta_{2}$ is $9.7 * 10^{6} \mathrm{MPa}$ G2 is $1 * 10^{6}$ MPa, $\eta_{2}$ is $3.3^{*} 10^{7} \mathrm{MPa} \cdot h$.The calculated parameters for the gob area long-term stability evaluation and similar engineering experimental evidences were provided for the long term stability evaluation.

\section{CONCLUSION}

a) the sandstone triaxial compression test results show that with the increase of load, there are three stages in the creep curve, the sample's total creep variable is big. Burgers model 
was used based on the test results, and puts forward the constitutive model of the parameters in the equation of $\left(\mathrm{K}, \mathrm{G}_{0}\right.$, $\eta_{1}, G_{1}, \eta_{2}$ ) values, which describes a site of Shaanxi sandstone long-term creep characteristics.

b) The Triaxial compression test which sandstone sample in 20KN under different confining pressure state of axial compression (1 MPa, $2 \mathrm{MPa}, 4 \mathrm{MPa}$ and $5 \mathrm{MPa}$ ) results show that when applying axial load of each level, sample usually produce obvious instantaneous deformation; the creep deformation tends to decay Under low stress level, a constant creep Under the high.

c)there have great influence to deformation which the size of the confining pressure in the creep process. With the increase of confining pressure, the axial creep deformation is reduced. It tells us that Axial rheological is not easy, mainly because of confining pressure have a kind of polymerization, distracted the axial pressure, lateral pressure on the rock. Creep rate change with the change of stress level and, when the gradient of stress at a fixed step by step growth, instantaneous deformation of rock creep is also gradually reduced. While stable deformation at a certain rate to maintain the same strain rate.

d) when the confining pressure load in $1 \mathrm{MPa}-2 \mathrm{MPa}$, the deformation increases in stability, and when the confining pressure reaches $4 \mathrm{MPa}$, the deformation of slope suddenly become smaller, the confining pressure of $4 \mathrm{MPa}, 5 \mathrm{MPa}$ sandstone deformation tends to be stable.

e) on the basis of the existing indoor triaxial compression test result, through computer numerical simulation, determine the parameter of Burgers model in triaxial compression test simulated by FLAC3D, the numerical simulation for the rheological test provides a reliable basis.

\section{REFERENCES}

[1] Zhang Z P, Wang Z Y, Peng H. Study on triaxial compression creep test and numerical simulation about mud rock in southern Shaanxi[J]. Hydrogeology \& Engineering Geology, 2011, 38(1):53-58

[2] Wang Yu, Li Jianlin, Liu Feng, and. Study on rheological behavior and rheological behavior of jointed silty sandstone [J]. rock and soil mechanics, 2012,12:3639-3644+3652.

[3] Zhu Jiebing, Wang Bin, Wu Aiqing. Jinping Hydropower Station Green sandstone three axis unloading experiment and nonlinear rheological damage creep constitutive model of [J]. Chinese Journal of rock mechanics and engineering, 2010,03:528-534.

[4] Yao Jun. study on creep characteristics of sandstone and its influencing factors [D]. Chengdu University of Technology, 2015

[5] [5] Zhu Changxing, Huang Zenghui, Han Wei. Study on nonlinear creep model of sandstone based on three axis creep test [J]. Journal of Henan Polytechnic University (NATURAL SCIENCE EDITION), 2015,04:468-471. (in Chinese)

[6] Li Liangquan, Xu Weiya,, Guo Yunqiang, et al. Long term strength evaluation of Xiangjiaba sandstone based on rheological test [J]. Cheng mechanics, 2010,11:127-136+143.

[7] static. Zia, Xie rock (rock) analysis of water science and engineering technology [J]. accelerated creep stage model, 2016,03:91-94.

[8] Zhu Changxing, Huang Zenghui, Han Wei. Study on nonlinear creep model of sandstone based on three axis creep test [J]. Journal of Henan Polytechnic University (NATURAL SCIENCE EDITION), 2015,04:468-471. (in Chinese)

[9] Gao Wenhua, Liu Zheng, and. Numerical simulation study on compression creep test of siltstone based on FLAC (3D) [J]. Chinese Journal of civil engineering, 2015,03:96-102. (in Chinese) 